\title{
Feedback Control in an Atomic Force Microscope Used as a Nano-Manipulator
}

\section{Hrouzek}

This paper offers a concise survey of the most commonly used feedback loops for atomic force microscopes. In addition it proposes feedback control loops in order to minimize the effect of thermal noise on measurements of weak forces, and to improve the manipulability of the AFM. Growing requirements to study and fabricate systems of ever-shrinking size mean that ever-increasing performance of instruments like atomic force microscopes (AFM) is needed. A typical AFM consists of a micro-cantilever with a sharp tip, a sample positioning system, a detection system and a control system. Present day commercial AFMs use a standard PI controller to position the micro-cantilever tip at a desired distance from the sample. There is still a need for studies showing the optimal way to tune these controllers in order to achieve high closed-loop positioning performance. The choice of other controller structures, more suitable for dealing with the robustness/performance compromise can also be a solution.

Keywords: automatic control, atomic force microscopy, thermal noise.

\section{Description of AFM}

Atomic Force Microscopy (AFM) is an aspect of Scanning Force Microscopy, and is capable of measuring the interaction force between the sample and a sharp tip mounted on the end of a weak cantilever, see Fig. 1. The cantilever is made of silicon and usually has a rectangular or triangular shape. Its length does not exceed $600 \mu \mathrm{m}$.

Two main AFM operation modes are used: contact mode and dynamic mode. In contact mode the cantilever is in full contact with the surface, and the interaction force (displacement of the lever) is determined by measuring the static deflection of the cantilever. This technique is capa- ble of detecting displacements on atomic scale resolution $(0.1-200 \mathrm{~nm})$. The dynamic detection mode can be divided into techniques called the tapping mode and the non(pseudo)- contact mode [1]. In these detection modes, the cantilever is driven, usually at its resonant frequency, with an amplitude typically less than $10 \mathrm{~nm}$. The cantilever driver is usually a piezo-electric element, but many experiments have been performed with electrostatic, magnetic, thermo-optic and acoustic coupling drivers. The driver is attached to the head of the microscope and the chip with the cantilever and tip is assembled on top of the driver.

In the tapping mode, the tip is touches the surface in each period of cantilever movement at its maximum deflection to-

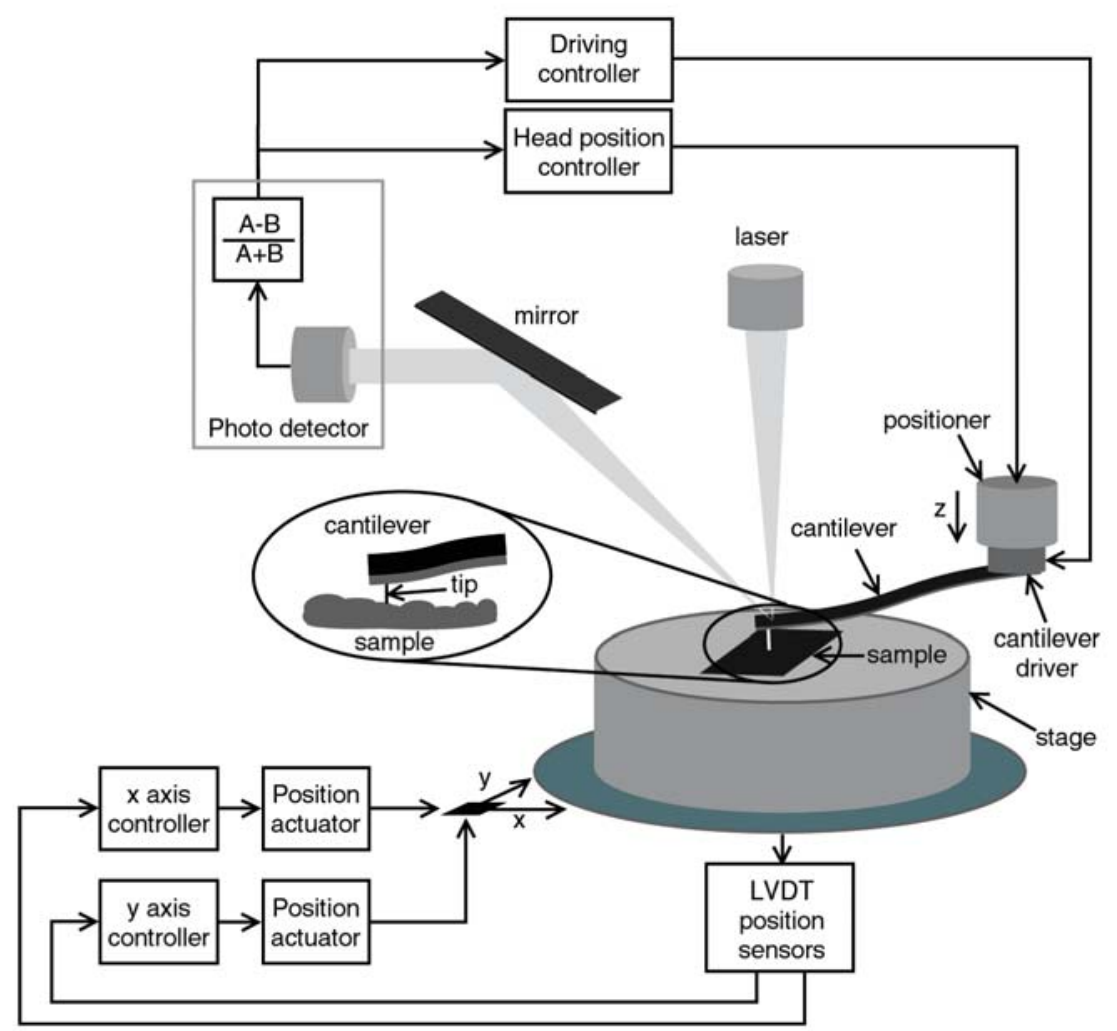

Fig. 1: Schema of an Atomic Force Microscope. Stage positioning is controlled by two loops (X-Y) displayed in the lower part of the picture. The driving and head positioning loops are at the top. Their only input is a signal from the photo detector that measures the deflection of the cantilever. 
wards the surface. There is direct mechanical contact between the tip and the surface. Another way to scan soft samples is with the use of a non-contact technique. The cantilever vibrates very close to the surface, but does not come into direct contact with it. Attractive long distance interaction forces affect the effective spring constant of the cantilever, and the cantilever shifts its resonance frequency. This technique is used for measuring weak forces, such as the van der Waals force, and for scanning soft samples.

Accuracy in measuring the cantilever displacement is very important in order to achieve good AFM resolution. Many techniques have been developed for this measurement, based on various principles, including capacitive and optical techniques as well as interferometry, optical beam deflection and laser diode detection. Each technique has specific advantages and disadvantages. A method widely used by many manufacturers of AFMs is the optical beam deflection technique, shown in Fig. 1. Its function is based on a sensitive photodetector which is able to detect very small laser beam displacements. The photodiode receiving the reflected laser beam from the cantilever is divided into two sections, $\mathrm{A}$ and $\mathrm{B}$. Due to the macroscopic length of the reflected light path, any deflection causes a magnified displacement of the reflected laser spot on the photodiode. The relative amplitudes of the signals from the two segments of the photodiode change in response to the motion of the spot. The difference signal $(\mathrm{A}-\mathrm{B})$ is very sensitive to cantilever deflection.

\section{Feedback control in the AFM}

Modern AFMs include many feedback loops to control different blocks of this complicated instrument. Some of the most exciting and still widely open areas in AFM feedback control are:

\subsection{Loop controlling position of the AFM stage on the $\mathrm{X}-\mathrm{Y}$ axis}

Many experiments have been performed with various techniques for moving the stage with the scanned sample with high precision and speed. Over many years, most AFM manufacturers and scientists have preferred piezo-electric stack actuators. These devices offer fine position capabilities achieving sub-Angstrom resolution and high-speed manipulation. Piezo electric actuators have disadvantages in nonlinear properties such as hysteresis and creep, which become more significant with the increasing need for fast movement given by the actuators, see Fig. 2. This illustrates the need for a better and more accurate description of the piezo elements and for improving the feedback control systems by robust design techniques.

Surface physicists have used AFM mainly for scanning purposes in the past. Recently, AFM has been used in many different fields of biology, medicine, material science, nanomanipulation and nano-lithography. These extended uses involve the need for an improvement in the accuracy and repeatability of movements with the tip. In addition, the possibilities of using AFMs as manipulators on an atomic scale rather than as classical scanners are being explored. The difference between these two approaches is displayed in Fig 3. The part on the left is a simple movement across the sample in straight lines, scanning. On the other side, we see the
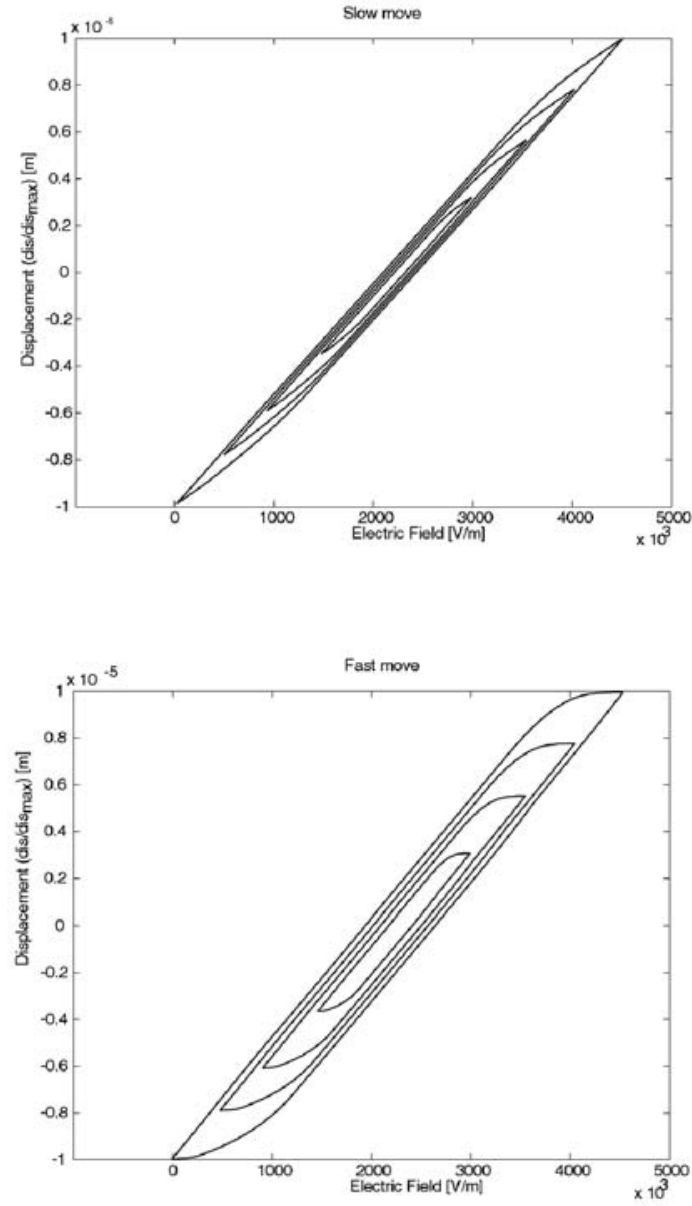

Fig. 2: Sketch of the hysteresis of a piezo stack actuator for slow and fast movement with the stage. Increasing speed of manipulation usually leads to problems with accuracy of positioning, due to the rising influence of nonlinearities such as hysteresis.

movement that is required to manipulate a small object on the surface. The cantilever has to reach the desired position, then approach the surface and make a movement which is not necessarily straight but can be of any shape. While manipulating an particle, it is very important to control the applied force by the tip on to the manipulated object.

Currently used regulators usually have a simple proportional-integral structure, which offers zero steady state regulation error but is not able to achieve the desired output in a short period of time. These regulators usually experience tracking problems that become significant with rising speed. The bandwidth limitations and non robust control are not satisfactory. Present-day feedback control loops for the $\mathrm{x}$ and $\mathrm{y}$

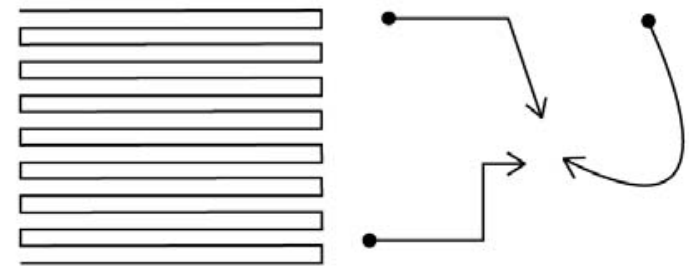

Fig. 3: Scanning approach on the left, manipulation approach on the right 

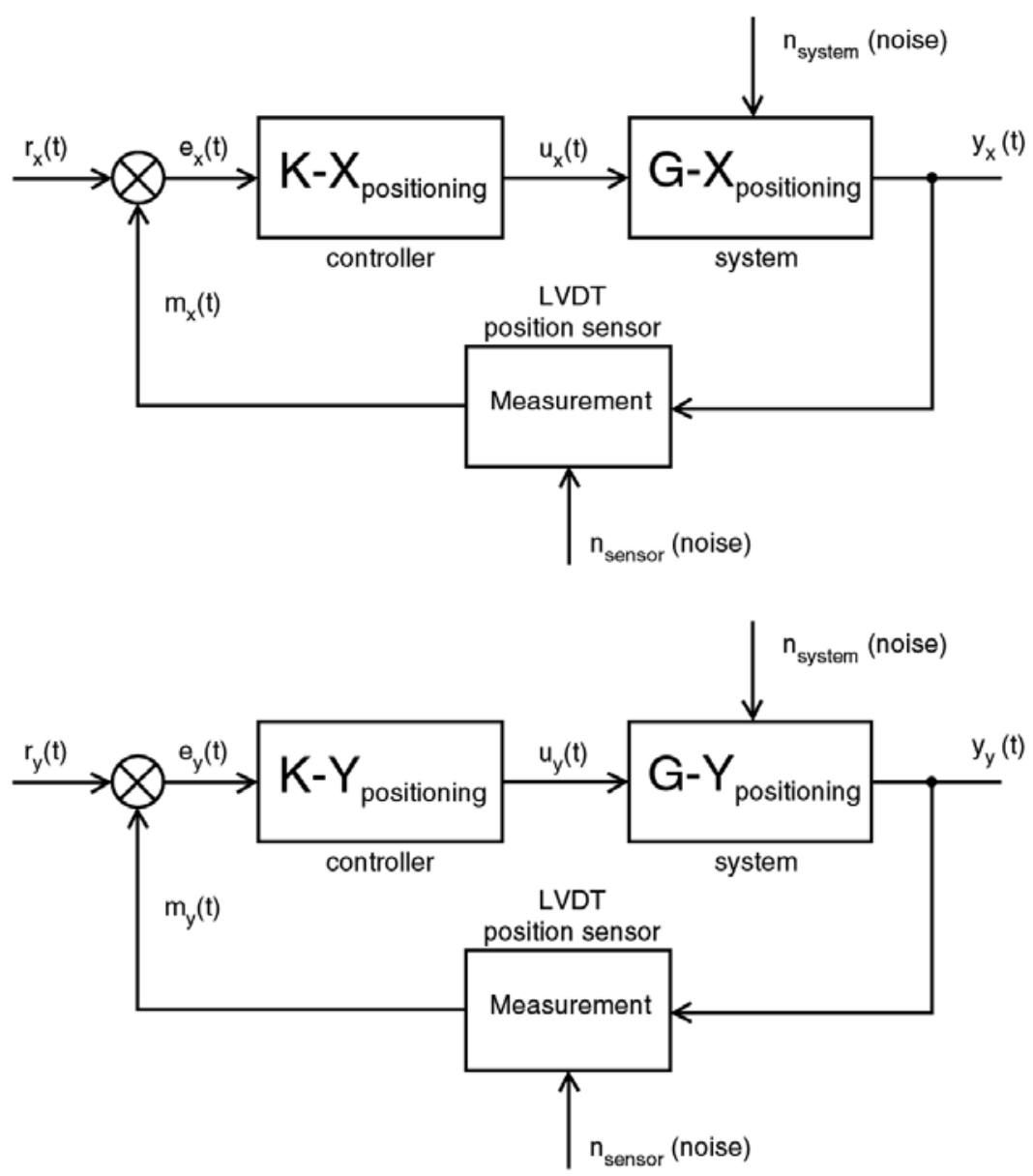

Fig. 4: Feedback control loop that can be used to control the position of the sample mounted on the stage of the AFM

axis have to be able to perform very complex movements at very high speed, rather than simply dragging the cantilever across the sample in straight lines with a frequency of a few hertz. This area is now open to new techniques and new ideas on how to control the nonlinearities of systems coming from piezo hysteresis in order to achieve the desired performance, see [2]. Fig. 4 displays the feedback control loops that are employed in the stage positioning system.

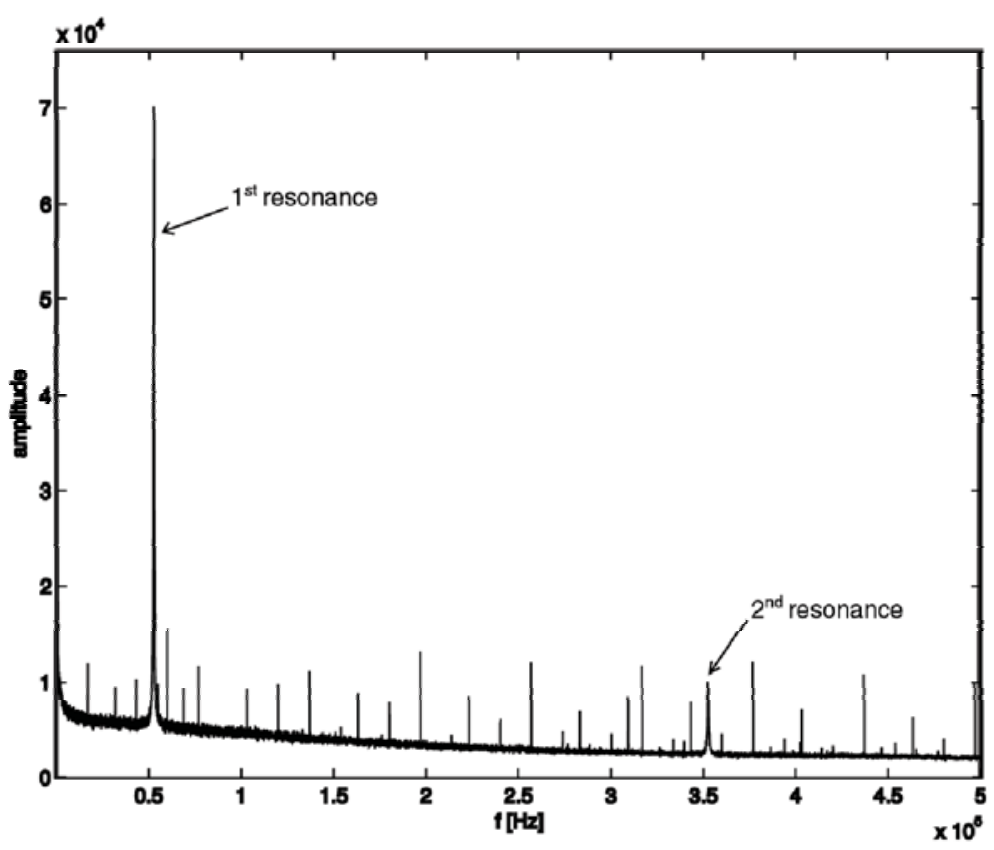

Fig. 5: Frequency spectrum of a freely vibrating cantilever driven by thermal noise (Brownian motion). The spectrum displays the first two resonance frequencies. Measurements on the NanoTec AFM 
Regulators have to be capable of treating nonlinearities of the controlled systems by using internal inverse models.

With increasing requirements for accuracy of stage positioning by the piezo actuator, the problem of measuring distances on a smaller scale is becoming apparent. Most applications use a Linear Variable Differential Transformer (LVDT), which offers the best sensitivity. Position sensors experience problems with exceedingly high levels of electric and thermal noise, which lower the resolution. This problem is another field in which of feedback control might be applied. An observer based regulator could achieve better signal noise separation than current systems without any feedback.

\subsection{Loop controlling the position of the AFM head on the $Z$ axis and cantilever excitation}

The description given below focuses on the non-contact operating mode of the AFM. The non-contact dynamic technique is very sensitive to thermal noise, but is frequently used for nondestructive measurement of soft biological samples.

The cantilevers are designed and manufactured small and soft enough not to destroy the surface of the measured sample. A very soft cantilever is sensitive to thermal excitation, which appears in the system as the largest source of noise [3]. Thermal noise significantly affects sensitivity, and limits possible improvements in resolution. Fig. 5 displays the amplitude spectrum measured on the cantilever without any artificial excitation. The spectrum shows little excitation across all frequencies, and large noise excitations are visible at the resonance frequencies of the cantilever. Some techniques already exist for improving sensitivity by eliminating the thermal noise of the cantilever. An interesting technique for improve is Noise Squeezing [4].
The cantilever is of course exposed to many other noises, such as electrostatic noise, electromagnetic noise and air turbulence. The influence of these noises can be eliminated by appropriate mechanical and electrical construction of the AFM. Only thermal noise cannot be attenuated by changes in the construction of the microscope. Some applications enable the sample and microscope to be put in a thermal bath at a very low temperature, which reduces the thermal excitation. However, there are many applications that have to be at room temperature. By applying a certain feedback control loop, the noise can be eliminated and the sensitivity of the instrument can be increased.

Scanning force microscopes are usually controlled by simple proportional-integral regulators which are not able to give the best possible results. Fig. 6 shows control loops affecting the sensitivity of the AFM. The regulators used in these loops need to be designed by optimal control techniques to achieve better sensitivity.

The control system consists of two main parts: the positioning loop and the driving loop. The positioning loop works at lower frequencies, and its task is to keep the head in a position that ensures a constant signal from the tip-surface interaction. It simultaneously measures the surface without losing any information. In scanning force microscopy this constant signal is known as the "set point", and ensures that the cantilever vibrates at its maximum harmonic deflection without hard tapping on the surface. The driving loop operates at the higher frequencies and keeps the cantilever excited at its resonant frequency and at the same amplitude despite the contact (van der Waals attractive and mechanical repulsive interactions) with the surface of the sample. This is usually done by the piezoelectric element (driver) harmonically vibrating at the desired frequency (slightly off the resonance frequency) with a very small amplitude.

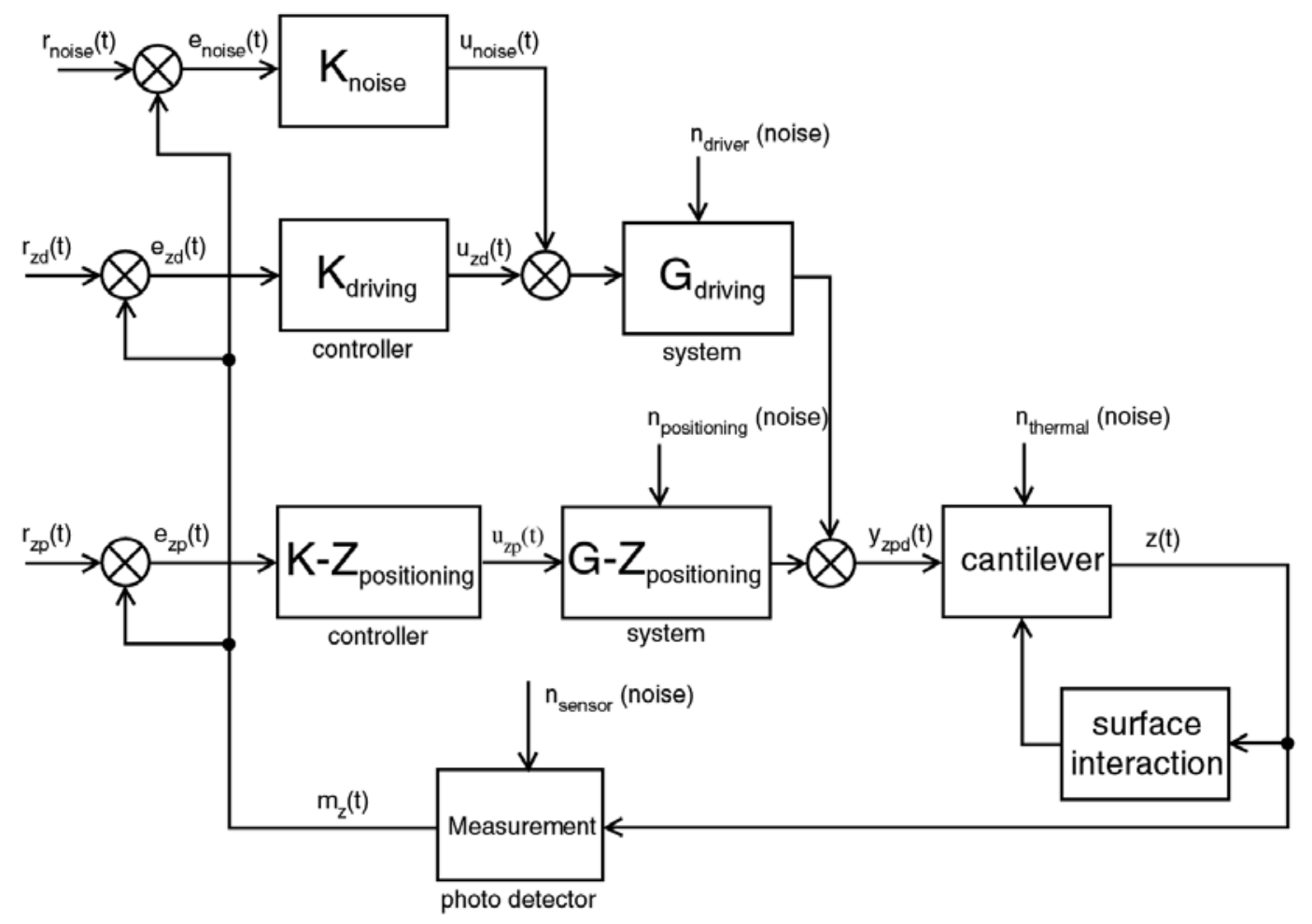

Fig. 6: Feedback control loops of the head positioning and the cantilever drive 
The third additional regulator is responsible for thermal noise attenuation. Application of this loop is very complicated, because the controller can easily destroy all measured signals by applying excessively large attenuating signals. Its function can be similar to Cold Damping techniques, known from cooling of nano-mechanical resonators or cavities [5]. The main goal of this technique is to stabilize the position of the mechanical part so that its displacement will be minimized and it appears virtually colder. In our case this is not fully true. The displacement caused by thermal noise needs to be eliminated, but the displacement caused by the driver or interaction forces needs to remain undisturbed, or less the measured signal will be changed. Another possible design is an observer based regulator, which would be able to approximate the influence of the thermal noise and interaction forces [6]. Both of these approaches face problems with determining which part of the signal is unwanted noise.

\section{Conclusion}

Atomic Force Microscopy is a new field for applying advanced control systems capable of improving the properties of the instrument. Presently used control systems are very simple, but ensure functionality. More sophisticated control system will enable Atomic Force Microscopes to be applied as nano-manipulators on an atomic scale, with improved sensitivity.

\section{Acknowledgments}

This work was supported by research project J22/98:26 2200012 "Research in Informatics and Control Systems".

\section{References}

[1] Stark, M., Stark, R. W., Heckl, W. M., Guckenberger, R.: "Spectroscopy of the Anharmonic Cantilever Oscillations in Tapping-Mode Atomic-Force Microscopy." Applied Physics Letters, November 2000.

[2] Salapaka, S., Sebastian, A., Cleveland, J. P., Salapaka, M. V.: "High Bandwidth Nanopositioner: A Robust Control Approach." Review of Scientic Instruments, September 2002.

[3] Cleland, A. N., Roukes, M. L.: "Noise Processes in Nanomechanical Resonators.” Journal of Applied Physics, 2002.

[4] Rugar, D., Grutter, P.: "Mechanical Parametric Amplication and Thermomechanical Noise Squeezing." Physical Review Letters, August 1991.

[5] Pinard, M., Cohadon, P. F., Briant, T., Heidmann, A.: "Full Mechanical Characterization Of A Cold Damped Mirror." Physical Review A, December 2000.

[6] Sebastian, A., Sahoo, D. R., Salapaka, M. V.: “An Observer Based Sample Detection Scheme for Atomic Force Microscopy. In Proceedings of the $42^{\text {nd }}$ IEEE Conference on Decision and Control, Maui, Hawaii, USA, December 2003.

Michal Hrouzek

e-mail:mhx@seznam.cz

Laboratoire d'Automatique de Grenoble

INP/UJF Grenoble, France and

Dept. of Control and Instrumentation

FEEC, BUT Brno,

Czech Republic 